\title{
Non-O-blood types associated with higher risk of high-grade atrioventricular block
}

Emrah Acar ${ }^{1}$, Servet İzci², Mehmet Inanir ${ }^{3}$, Mehmet Fatih Yılmaz ${ }^{4}$, Alev Kılıçgedik², Yeliz Güler ${ }^{5}$, Ibrahim Akin Izgi², Cevat Kirma²

\author{
${ }^{1}$ Department of Cardiology, Gumushane State Hospital, Gumushane, Turkey \\ ${ }^{2}$ Kartal Koşuyolu Heart and Vascular Disease Research and Training Hospital, \\ Istanbul, Turkey \\ ${ }^{3}$ Department of Cardiology, Bolu Abant Izzet Baysal University, Bolu, Turkey \\ ${ }^{4}$ Department of Cardiology, Yedikule Chest Disease and Chest Surgery Research \\ and Training Hospital, Istanbul, Turkey \\ ${ }^{5}$ Department of Cardiology, Kilis State Hospital, Kilis, Turkey
}

Submitted: 17 July 2019

Accepted: 15 September 2019

Arch Med Sci Atheroscler Dis 2019; 4: e243-e247

DOI: https://doi.org/10.5114/amsad.2019.90072

Copyright $\odot 2019$ Termedia \& Banach

\section{A bstract}

Introduction: The non-O phenotype of the ABO genotype has been linked with an increased risk of cardiovascular disease. Atrioventricular (AV) block (AVB) is defined as retardation or cessation in the route of an electrical impulse passing from the atria to the ventricles because of an anatomical or functional disruption in the conduction system. We aimed to interpret the association between blood group status and high-grade atrioventricular block (HAVB).

Material and methods: This study was implemented as a retrospective review of the recorded data of patients diagnosed with high-grade AV block and a control group. The study population consisted of 640 patients with HAVB and 570 control subjects.

Results: Presence of non-O blood group $(p<0.001)$ was significantly more prevalent in HAVB patients than in the control subjects. Blood group type was the sole independent predictor of HAVB in multiple regression analysis $(p<0.001, \mathrm{OR}=1.35,95 \% \mathrm{Cl}: 1.08-1.57)$. Also, third-degree AVB had a higher incidence in the non-O blood subgroup and also non-O blood group was a predictor of third-degree AVB $(p<0.001, \mathrm{OR}=1.39,95 \% \mathrm{Cl}: 1.13-1.69)$. The incidence of HAVB did not distinguish between the two Rh (D) groups. Rh (D) status did not have an impact on HAVB.

Conclusions: This is the first study that has evaluated the potential relationship between HAVB and ABO blood groups. The main finding of this report is that patients with non-O blood group types have a higher risk for development of HAVB compared with O blood group patients.

Key words: atrioventricular block, blood type, fibrosis, genotype.

\section{Introduction}

Atrioventricular (AV) block (AVB) is defined as retardation or cessation in the route of an electrical impulse passing from the atria to the ventricles because of an anatomical or functional disruption in the conduction system. The conduction impairment can be temporary or permanent, with conduction that is delayed, intermittent, or absent. There are several types of AVB such as first-degree AVB, second degree AVB (Mobitz type-1

\author{
Corresponding author: \\ Emrah Acar MD \\ Department \\ of Cardiology \\ Gumushane State Hospital \\ Gumushane, Turkey \\ Phone: +90 5358819708 \\ Email: dreacar44@hotmail. \\ com
}


and type-2), third degree AVB, and advanced AVB. Commonly, AVBs other than first-degree AVB are accepted as high-grade AVB (HAVB) (i.e., necessary to evaluate for causes and treatment). The potential etiologies of HAVB are quite numerous and encompass reversible causes (both pathologic and iatrogenic) as well as idiopathic causes. Common potentially reversible causes include pathologic (acute or chronic myocardial ischemia, cardiomyopathy, myocarditis, endocarditis with abscess formation, hyperkalemia, hypervagotonia, etc.) and iatrogenic (medication-related such as AV nodal blocking medications, post-cardiac surgery, post-catheter ablation, post-transcatheter aortic valve implantation).

Other pathologic causes may be progressive or irreversible (e.g., infiltrative malignancies, neuromuscular diseases). However, in half or more of the cases, no specific reversible causes are identified, and the block is assumed to be secondary to the idiopathic progressive cardiac conduction disease with myocardial fibrosis and/or sclerosis that disturbs the conduction system. Congenital complete heart block is generally irreversible.

The $A B O$ blood group is defined by the presence of $A$ and $B$ antigens on the superficial membranes of the red blood cells (RBCs). Furthermore, these antigens are widely embodied on the membranes of a wide range of cells, including platelets, vascular endothelium, and epithelium as well as in body fluids (such as in saliva). ABO blood groups have been related to various disease phenotypes, especially cardiovascular diseases. The most common etiologic causes of death in developed countries are cardiovascular diseases, and their prevalence rate is rapidly rising and evolving in developing countries. There have been considerable historical interrelations between non-O blood group status and an increase in some cardiovascular disorders. Recent genetic studies have described $A B O$ as a locus for thrombosis, myocardial infarction, and multiple cardiovascular risk biomarkers, directing attention to mechanisms and potential for clinical advances. Progress in our comprehension of the physiologic enormity of various endothelial and platelet-derived circulating glycoproteins is illuminating the mechanisms through which the $A B O$ blood group may identify overall cardiovascular disease risk. The role of blood group antigens in the pathogenesis of various cardiovascular disorders remains a captivating issue with the potential to lead to novel therapeutics and prognostics and to diminish the global burden of cardiovascular diseases.

Recently, there have been several reports showing that blood groups may play a role as an independent genetic risk factor predicting accelerated fibrosis. So, we hypothesize that blood types may also be a predisposing factor for idiopathic HAVB which is associated with myocardial fibrosis and/ or sclerosis.

\section{Material and methods}

This study was carried out as a retrospective analysis of the records of patients diagnosed with high-grade AV block in the emergency service and outpatient clinics at Kosuyolu Cardiovascular Research and Training Hospital and Gumushane State Hospital via the hospital information management system. Informed consent forms were taken from all of the patients and control subjects. Ethical approval was obtained from the institution's Ethics and Research Committee. Inclusion criteria were < 65 years old, non-congenital AV block, idiopathic HAVB, and a public ABO-Rh blood group. Patients with reversible cause-related HAVB (ischemia, cardiomyopathy, drug-induced, metabolic and electrolyte imbalance, iatrogenic) were excluded from the study. Age, sex, ABO-Rh blood groups, hypertension, diabetes mellitus, hyperlipidemia, atrial fibrillation, smoking, New York Heart Association (NYHA) class, stroke/transient ischemic attack, non-central nervous system embolism, and coronary artery disease were recorded. The distribution of ABO-Rh blood groups among HAVB patients was compared with Kosuyolu Cardiovascular Research and Training Hospital blood donors with normal atrioventricular conduction or first-degree atrioventricular block.

\section{Laboratory analysis}

ABO-Rh blood groups were defined by the lam agglutination technique using a blood grouping reagent (Dia-Gast, Loos, France), the microplate agglutination procedure using the Galileo System (Stratec, Frankfurt, Germany), or gel centrifugation assay using the $\mathrm{IH}-1000$ Fully Automated System (DiaMed, Cressier, Switzerland).

\section{Electrocardiographic evaluation}

Second-degree (Mobitz type-1 and Mobitz type-2) and third-degree AV block, with or without accompanying bundle branch block, were considered high-degree AV block, and all arrhythmic events were arbitrated by a central committee.

\section{Statistical analysis}

Data are shown as mean \pm SD for continuous variables and as proportions for categorical variables. Differences between proportions were analyzed using the $\chi^{2}$ test and substituted by the Fisher exact test when the expected cell count was $<5$. Student's $t$-test analyzed differences between mean values. Variables with a $p$-value $\leq 0.1$ were 
selected for logistic regression analysis. In order to describe any independent associates of HAVB, a logistic regression analysis was conducted. Statistical examinations were performed using SPSS version 13.0 (SPSS Inc., Chicago, Illinois). A twotailed $p$-value of $<0.05$ was considered significant for all analyses.

\section{Results}

The study population ( $n=1210 ; 64.8 \%$ female) involved patients with HAVB $(n=640)$ versus the control group ( $n=570$ ) with a normal AV conduction time and first degree AVB. Blood group $O$ was the most common phenotype (35.1\%) followed by blood group A (24.5\%), B (22.5\%), and AB (17.9\%). The majority of patients (65.7\%) were Rh (D) positive, whereas $34.3 \%$ were Rh (D) negative. The detailed baseline patient characteristics are shown in Table I. Third-degree AVB (48.7\%) was the most common HAVB type, followed by second-degree Mobitz type-1 AVB (32.3\%) and second-degree Mobitz type-2 AVB (19.0\%) (Table II). In the control group, normal AV conduction was $62.4 \%$ and in the remaining part of the control group was first-degree AVB (37.6\%). Presence of a non-O blood group ( $p<0.001)$ was significantly more prevalent in HAVB patients than in the control subjects. Blood group type was the sole independent predictor of HAVB on multiple regression analysis ( $p<0.001, \mathrm{OR}=1.3,95 \% \mathrm{Cl}: 1.08-1.57$ ) (Table III). Also third-degree AVB had a higher incidence in non-O blood subgroup and also non-O blood group was a predictor of third-degree AVB $(p<0.001, \mathrm{OR}=1.39,95 \% \mathrm{Cl}: 1.13-1.69)(\mathrm{Ta}-$ ble IV). The incidence of HAVB did not differ significantly between the two Rh (D) groups. Rh (D) status has no impact on HAVB.

\section{Discussion}

The ABO blood group is established by the presence of $A$ and $B$ antigens on the surface membranes of the red blood cells (RBCs). Additionally, these antigens are widely present on the membranes of a vast cluster of cells, including platelets, vascular endothelium, and epithelium [1]. Moreover, they are secreted in saliva and body fluids [2].
Table I. Baseline characteristics of the study groups

\begin{tabular}{|lccc|}
\hline Parameter & $\begin{array}{c}\text { HAVB } \\
(n=640)\end{array}$ & $\begin{array}{c}\text { Control } \\
(n=570)\end{array}$ & $P$-value \\
\hline Demographic: & & & \\
\hline Gender, female/male & $422 / 218$ & $362 / 208$ & 0.234 \\
\hline Age [year] & $57 \pm 10.8$ & $53.9 \pm 11.1$ & 0.198 \\
\hline \begin{tabular}{l} 
Blood group type: \\
\hline O/non-O group
\end{tabular} & $292 / 346$ & $253 / 317$ & $<0.001$ \\
\hline Rh positive & 402 & 394 & 0.598 \\
\hline \begin{tabular}{l} 
Medical history: \\
\hline Hypertension
\end{tabular} & 219 & 231 & 0.548 \\
\hline $\begin{array}{l}\text { Diabetes mellitus } \\
\text { Atrial fibrillation }\end{array}$ & 31 & 29 & 0.687 \\
\hline \begin{tabular}{l} 
NYHA III-IV \\
\hline Stroke or TIA
\end{tabular} & 8 & 32 & 0.79 \\
\hline \begin{tabular}{l} 
Hyperlipidemia \\
\hline Smoking
\end{tabular} & 242 & 240 & 0.621 \\
\hline \begin{tabular}{l} 
Non-CNS embolism \\
\hline $\begin{array}{l}\text { Coronary artery } \\
\text { disease }\end{array}$
\end{tabular} & 234 & 209 & 0.841 \\
\hline
\end{tabular}

Table II. HAVB types and incidence in the disease group

\begin{tabular}{|lc|}
\hline HAVB type & Percent of patient (\%) \\
\hline Third degree AVB & 48.7 \\
\hline $\begin{array}{l}\text { Second degree Mobitz } \\
\text { type 1 }\end{array}$ & 32.3 \\
\hline $\begin{array}{l}\text { Second degree Mobitz } \\
\text { type 2 }\end{array}$ & 19 \\
\hline
\end{tabular}

Several previous studies have revealed that individuals with the non-O blood group had higher rates of cardiovascular events. Rumley et al. reported that patients with a non-O blood group have lower plasma levels of factor VIIIC and von Willebrand factors than the patients with blood group $\mathrm{O}$; therefore thrombotic disease varies among them [3]. Kole described the relation be-

Table III. Predictors of HAVB in the HAVB patient group

\begin{tabular}{|lccc|}
\hline Parameter & Univariate $p$-value & Multivariate $p$-value & OR (95\% Cl) \\
\hline Non-O blood group & $<0.001$ & $<0.001$ & $1.35(1.08-1.57)$ \\
\hline Age & 0.423 & 0.283 & $0.958(0.917-1.014)$ \\
\hline Female sex & 0.161 & 0.069 & $1.017(1.005-1.031)$ \\
\hline Hypertension & 0.479 & 0.198 & $1.247(0.673-2.342)$ \\
\hline Coronary artery disease & 0.198 & 0.139 & $1.437(0.859-2.439)$ \\
\hline
\end{tabular}


Table IV. Predictors of third-degree AVB in the HAVB patient group

\begin{tabular}{|lccc|}
\hline Variable & Univariate $p$-value & Multivariate $p$-value & OR $(95 \% \mathrm{Cl})$ \\
\hline Non-O blood group & $<0.001$ & $<0.001$ & $1.39(1.13-1.69)$ \\
\hline Age & 0.308 & 0.298 & $1.009(0.879-1.312)$ \\
\hline Female sex & 0.129 & 0.092 & $0.903(0.829-1.304)$ \\
\hline
\end{tabular}

tween $\mathrm{ABO}$ blood groups and cardiovascular disease risk profile in a general population-based meta-analysis [4]. Koster et al. showed that the risk of thrombosis rose with increasing VWF or factor VIII concentration and was higher in subjects of non-O blood groups than in those of group $\mathrm{O}[5]$. Also, in another paper it was reported that patients with non-O blood groups have increased risk for venous thromboembolism [6, 7]. In a study which included patients with mechanical prosthetic valve thrombosis, Astarcioğlu et al. detailed that non-O blood group was an independent risk factor for prosthetic valve thrombus [8]. In the Framingham study population, peripheral vascular disease was more prevalent in patients with a non-O blood group [9]. Koster et al. revealed that pulmonary embolism is more common in patients with a non-O blood group [5], and Clark et al. reported that the $A B O$ blood group influenced the pathophysiology and prognosis in patients with cerebral ischemia of arterial origin [10].

Using genome-wide association studies (GWAS) in recent years has encouraged immense breakthroughs across almost the whole spectrum of cardiovascular diseases [11]. GWAS have confirmed $A B O$ as a locus for venous thromboembolism (VTE), myocardial infarction (MI), and multiple cardiovascular biomarkers [11].

Also, it is detailed that the $A B O$ locus may also play a role in the fibrotic/sclerotic process [11]. Recently there are reports about blood types and diseases in which there is a fibrotic process. Hakyemez et al. reported that blood types could play a role as a genetic risk factor for severe hepatic fibrosis and progression to cirrhosis [12]. Nikam et al. described that people with blood group $B$ were at higher risk of developing oral submucous fibrosis as compared to other groups [13]. There is also some relationship with blood groups and fibrosis in cancer [14-16]. To our best knowledge, there has been no evidence about cardiac conduction system fibrosis with blood groups until now.

Atrioventricular (AV) block is defined as retardation or interruption in the route of an electrical impulse from the atria to the ventricles due to an anatomical or functional impairment in the conduction system [17]. The conduction impairment can be temporary or permanent, with conduction that is delayed, intermittent, or absent. In nearly all cases, the diagnosis of high-grade AV block can be made in a patient with suggestive symptoms (e.g., fatigue, dyspnea, presyncope, and/or syncope) by obtaining a surface electrocardiogram (ECG), ideally a full 12-lead ECG but sometimes a single-lead rhythm strip is adequate if a full 12lead ECG cannot be obtained. For the rare patient with a non-diagnostic surface ECG, invasive electrophysiological studies can definitively diagnose high-grade AV block and accurately identify the level of the block [17].

The potential etiologies of HAVB are quite numerous and encompass reversible causes (both pathologic and iatrogenic) as well as idiopathic causes. Common potentially reversible causes in clude pathologic (acute or chronic myocardial ischemia, cardiomyopathy, myocarditis, endocarditis with abscess formation, hyperkalemia, hypervagotonia, etc.) and iatrogenic (medication-related such as AV nodal blocking medications, post-cardiac surgery, post-catheter ablation, post-transcatheter aortic valve implantation) [18].

Other pathologic causes may be progressive or irreversible (e.g., infiltrative malignancies, neuromuscular diseases). However, the most common cause of acquired conduction system disease is idiopathic fibrosis [19]. There are two well-known forms of acquired idiopathic conduction disorder in the elderly population. First, Lev's disease results in sclerosis of the left side of the cardiac skeleton, and affects the branching His bundle [20]. Second, Lenegre's disease is thought to be a hereditary genetic disorder that can affect middle-aged people. This degenerative process involves the more distal portions of the bundle branches [21]. Both Lev's and Lenegre's diseases cause right bundle branch block and left anterior hemiblock in people without other cardiac abnormalities, and can eventually progress to complete heart block.

Since blood groups may play a role in the fibrosis process, we advanced the hypothesis that blood groups can also have a role in fibrosis associated with idiopathic atrioventricular block.

The main limitation of the study is that our study population is small. In future it can be evaluated in a larger study population.

In conclusion, this is the first study to evaluate the potential relationship between HAVB and $A B O$ blood groups. We try to answer the question whether the relationship of blood groups with fibrosis in other system diseases is also efficient in 
fibrosis associated with idiopathic atrioventricular block. The main finding of this report is that patients with non-O blood group types have a higher risk for development of HAVB compared with O blood group patients.

\section{Conflict of interest}

The authors declare no conflict of interest.

\section{References}

1. Oriol R, Mollicone R, Coullin P, et al. Genetic regulation of the expression of $A B H$ and Lewis antigens in tissues. APMIS Suppl 1992; 100: 28-38.

2. Hosoi E. Biological and clinical aspects of $A B O$ blood group system. J Med Investig 2008; 55: 3-4.

3. Rumley A, Lowe GD, Sweetnam PM, et al. Factor VIII, von Willebrand factor and the risk of major ischaemic heart disease in the Caerphilly Heart Study. Br J Haematol 1999; 105: 110-6.

4. Kole TM. ABO blood group and cardiovascular outcomes in subjects from the general population: a meta-analysis. Heart Failure 2017. April 30, 2017. Paris, France.

5. Koster T, Blann AD, Briet E, et al. Role of clotting factor VIII in effect of von Willebrand factor on occurrence of deep-vein thrombosis. Lancet 1995; 345: 152-5.

6. Procare-GEHT Group. ABO blood group but not hemostasis genetic polymorphisms significantly influence thrombotic risk: a study of 180 homozygotes for the Factor V Leiden mutation. Br J Haematol 2006; 135: 697-702.

7. Tirado I, Mateo J, Soria JM, et al. The ABO blood group genotype and factor VIII levels as independent risk factors for venous thromboembolism. Thromb Haemost 2005; 93: 468-74.

8. Astarcıoğlu MA, Kalçık $M$, Yesin $M$, et al. ABO blood types: impact on the development of prosthetic mechanical valve thrombosis. Anatol J Cardiol 2016; 16: 820-3.

9. Garrison RJ, Havlik RJ, Harris RB, et al. ABO blood group and cardiovascular disease the Framingham Study. Atherosclerosis 1976; 25: 311-8.

10. Clark P, Meiklejohn DJ, O'Sullivan A, et al. The relationships of ABO, Lewis and Secretor blood groups with cerebral ischemia of arterial origin. J Thromb Haemost 2005; 3: 2105-8.

11. Zeller T, Blankenberg S, Diemert P. Genomewide association studies in cardiovascular disease - an update 2011. Clin Chem 2012; 58; 92-103.

12. Hakyemez IN, Durdu B, Bolukcu S, et al. Evaluation of the relationship between $\mathrm{ABO} / \mathrm{Rh}$ blood groups and severity of liver fibrosis in patients with chronic hepatitis B. Viral Hepatitis J 2016; 22: 23-7.

13. Nikam P, Bommanavar S, Malik S, et al. Evaluation of the $\mathrm{ABO}$ blood group system in patients suffering from oral sub mucous fibrosis. Int J Curr Res 2015; 7: 13295-8.

14. Rummel S, Shriver CD, Rachel, Ellsworth RE. Relationships between the ABO blood group SNP rs505922 and breast cancer phenotypes: a genotype-phenotype correlation study. BMC Med Genet 2012; 13: 41.

15. Tyagi SP, Pradhan S, Agarwal P. Blood groups in malignant diseases. J Indian Med Assoc 1965; 45: 645-50.

16. Jovanvic-Cupic S, Blagogevic J. ABO histoblood groups and $\mathrm{Rh}$ system in relation to malignant tumors of the digestive tract in Bosnia and Herzegovina. Arch Biol Sci 2008; 60: 593-9.

17. Kusumoto FM, Schoenfeld MH, Barrett C, et al. 2018 ACC/AHA/HRS Guideline on the evaluation and management of patients with bradycardia and cardiac conduction delay. Circulation 2019; 140; e382-482.

18. Brignole M, Auricchio A, Baron-Esquivias G, et al. 2013 ESC Guidelines on cardiac pacing and cardiac resynchronization therapy: the Task Force on cardiac pacing and resynchronization therapy of the European Society of Cardiology (ESC). Developed in collaboration with the European Heart Rhythm Association (EHRA). Eur Heart J 2013; 34: 2281-2329.

19. Yu CM, Chan JY, Zhang Q, et al. Biventricular pacing in patients with bradycardia and normal ejection fraction. N Engl J Med 2009; 361: 2123-34.

20. Rosen KM, Dhingra RC, Loeb HS, Rahimtoola SH. Chronic heart block in adults. Clinical and electrophysiological observations. Arch Intern Med 1973; 131: 663-72.

21. Curtis AB, Worley SJ, Adamson PB, et al. Biventricular pacing for atrioventricular block and systolic dysfunction. N Engl J Med 2013; 368: 1585-93. 